\title{
A glimpse of life science research at Tsinghua
}

\author{
SHI YiGong \\ School of Life Sciences, Tsinghua University, Beijing 100084, China
}

Received November 15, 2013; published online December 23, 2013

Citation: Shi YG. A glimpse of life science research at Tsinghua. Sci China Life Sci, 2014, 57: 1-3, doi: 10.1007/s11427-013-4594-x

Life Science at Tsinghua University has a long and eventful history. The Biology Department, founded in 1926, was among the oldest and most prestigious in China. In 1952, the Biology Department, together with other natural science departments, were eliminated from the University by order of the central government. In 1984, the Department of Biological Science and Biotechnology was re-established, with Professor Poo Mu-ming being the founding Director. In the ensuing 25 years, the Department went through rapid transformations and gained national and international recognition. In September 2009, the Department of Biological Science and Biotechnology was replaced by the School of Life Sciences.

Life Science, the pillar of healthcare and medicine, is at the forefront of modern research in the 21 st century. Motivated by the goal of becoming a world-class university, Tsinghua has placed a top priority on life sciences for development and expansion since 2006. Consequently, the physical space has increased by approximately 300\%, and the number of independent laboratories in life sciences has nearly tripled. At present, there are approximately 120 independent principal investigators (PIs) in the School of Life Sciences and School of Medicine. These include approximately 33 tenured Full Professors, and 87 tenure-track Assistant and Associate Professors. These PIs include five academicians of the Chinese Academy of Sciences/ Engineering, 11 members of the Thousand Talent Program, 22 Cheung Kong Scholars of the Ministry of Education, and 21 Outstanding Young Talents of the National Natural Science Foundation. These PIs are pursuing innovative re-

email: shi-lab@tsinghua.edu.cn search in a wide range of disciplines including but not limited to biochemistry, biomedical engineering, biophysics and structural biology, cell and molecular biology, genetics and development, molecular immunology, neuroscience, plant biology, system biology, synthetic biology, and other traditional and emerging research areas.

This special issue includes 16 original research articles from the laboratories of 17 independent PIs. These articles, covering a broad range of topics, reflect the latest research discoveries from the life science community at Tsinghua. I would like to briefly introduce these PIs and their research contributions reported in this special issue.

Research in Dr. Chen GuoQiang's laboratory focuses on microbial polyhydroxyalkanoates (PHAs) metabolic engineering and PHA biomaterials application. The current study [1] reports controllable composition of poly(3hydroxyalkanoate) (PHA) consisting of 3-hydroxydodecanoate (3HDD) and phenyl group on the side-chain with edited chromosome of Pseudomonas entomophila to weaken its $\beta$-oxidation. The mechanical and thermal properties of this new material varied dramatically depending on the monomer ratios. These results demonstrate the potential of designing and making novel functional PHA using chromosome editing in Pseudomonas entomophila.

Dr. Zhang DaPeng is interested in understanding the functional mechanism of phytohormone abscisic acid (ABA), a key hormone in plant adaptation to adverse environment. His group previously identified the chloroplast $\mathrm{Mg}$-chelatase $\mathrm{H}$ subunit as a putative ABA receptor (ABAR) in Arabidopsis thaliana, which antagonizes a group of WRKY-domain transcription repressors to relieve the inhibition of ABA-responsive genes. In the present report [2], 
they show that the chloroplast co-chaperonin CPN20, an ABAR interaction protein, represses the ABAR-WRKY40coupled pathway, and ABA relieves the repression by inhibiting the ABAR-CPN20 interaction.

Dr. Lu Zhi John's group aims to understand how information is encoded in the DNA and RNA sequences and how they regulate one another in a biological system. The main ongoing effort focuses on characterization of novel non-coding RNA genes. In the current report [3], they developed a bioinformatics framework to analyze transcriptome-wide RNA-protein interactions, focusing on their structural context. Their investigation gives rise to an ensemble pattern of RNA-binding-protein binding sites in the structured non-coding regions of a eukaryotic transcriptome.

Research from Dr. Qi YiJun's laboratory focuses on dissecting the mechanisms of RNAi pathways and the biological functions of small RNAs in plants. His laboratory discovered miRNAs in unicellular green algae and a novel class of DSB-induced small RNAs (diRNAs) in plants and humans. In this paper [4], they report identification of miRNAs and analyses of their targets in a multicellular green algae, Volvox carteri. This catalog of miRNAs and their targets provides a resource for further studies on the evolution, biological functions, and genomic properties of miRNAs in green algae.

Dr. Zhong Yi's laboratory is interested in studying molecular and circuit mechanisms underlying memory and forgetting in Drosophila. The major approach is to study genes identified in human disorders with a component of cognitive dysfunctions. The article presented in this issue [5] is an extension of their work on schizophrenia susceptibility gene, dysbindin. It shows that one mechanism contributing to Dysbindin-dependent regulation of dopamine activity, but not glutamatergic synaptic transmission, is confined to glial cell through regulation of activity of a monoamine synthase, Ebony.

Dr. Zhang XueGong's research interests include development of methods for processing and analyzing highthroughput biology data especially NGS data, and the analysis and discovery of hidden patterns in biology data. The current manuscript [6] took the extensively studied yeast antisense profile to probe whether sequence features around protein-coding genes can predict the existence of partner antisense transcripts. The experimental results show that there are imperfect but significant signals in the sequence composition features around genes that are informative for predicting antisense transcripts.

Dr. Yu Li's laboratory works on autophagy, with a focus on understanding the molecular machinery of autophagy in the context of broader cellular membrane trafficking system and the regulation of autophagy in the framework of metabolism regulatory network. In this work [7], they show that the autophagy essential gene Atg5 is required for biogenesis of late endosomes and lysosomes in an autophagy- independent manner, thus revealing an unexpected role for Atg5 and shedding lights on late endosome and lysosome biogenesis.

Research from Dr. Wu JiaWei's laboratory focuses on understanding the regulatory mechanisms of particular molecules in metabolic and developmental signaling pathways using biochemical, structural, molecular and cellular methods. In the current study [8], they report the crystal structure of the homotypic PB1-PB1 complex between $\mathrm{PKC} \zeta$ and $\mathrm{p} 62$ that is critical for NF- $\mathrm{KB}$ signaling. Further structural and biochemical analyses unravel the molecular mechanism of aurothiomalate inhibition on the $\mathrm{PKC \zeta}$-p62 interaction, which serves as the basis for designing inhibitors of rheumatoid arthritis.

Dr. Qi Hai aims to better understand regulation of humoral immunity with a particular focus on dynamic regulation of $\mathrm{T}$ and $\mathrm{B}$ lymphocyte interactions and germinal center formation in vivo. Dr. Qi previously showed that SAP specifically controls the strength of cognate T-B interactions. In the current paper [9], Dr. Qi and his colleagues report serendipitous identification of a new isoform of murine SAP gene and its expression and functional characterization.

Dr. Zhang LinQi's primary research interest is HIV pathogenesis, with a focus on virologic and immunologic changes during the course of disease and treatment with highly active antiretroviral therapy. The main objective of the current study [10] is to characterize neutralizing antibody resistant HIV-1 against VRC01, a broadly neutralizing monoclonal antibody identified in an elite controller. The results indicate these strains are present and continue to evolve during antiretroviral therapy. Persistence and continuous evolution of VRC01-resistant HIV-1 in vivo will impose greater challenge to our future preventative and therapeutic interventions based on VRC01.

Dr. Wang XinQuan is interested in the recognition of extracellular stimuli by cell surface receptors. Dr. Chang ZhiJie is dedicated in the study of molecular mechanisms controlling human diseases including cancers and rheumatoid arthritis. CREPT and p15RS are two homologous proteins with opposing functions on cell proliferation. In this paper [11], Drs. Wang and Chang collaborated to elucidate the respective roles of N-terminal RPR and C-terminal domains of CREPT and p15RS in recognizing RNA polymerase II by a combination of structural and biochemical methods. The results also reveal structural basis that may underlie the functional difference between these two homologous proteins.

Dr. Li Peng focuses her research on the fusion and growth of lipid droplet, an important sub-cellular organelle responsible for lipid storage, and its role in obesity development and fatty liver formation. They have shown here [12] that Cidea, a protein known to control energy homeostasis and obesity development, plays a crucial role in controlling lipid droplet fusion and lipid storage in brown and white fat tissue. Their research reveals a novel regulatory pathway in 
obesity development and insulin sensitivity.

Dr. Chen YingHua is interested in the mechanistic studies of HIV-1 entry and the development of broad-spectrum anti-viral inhibitors and vaccines. This study [13] reports induction of the 10E8-like neutralizing antibodies against HIV-1 by a pre-designed conformational peptide derived from 10E8 epitope. Thus, structure-specific peptide immunogen derived from 10E8 epitope could be a promising new concept for HIV-1 vaccine development.

Dr. Chen YeGuang's laboratory aims to understand how TGF- $\beta$ and Wnt signaling are regulated and to elucidate their functions in embryogenesis, stem cell self-renewal and differentiation, tissue fibrosis and tumorigenesis. In this study [14], the nuclear protein YY1 was identified to associate with Smad7, a negative regulator of TGF- $\beta$ signaling. These two proteins cooperate to antagonize TGF- $\beta /$ Smaddriven transcription by recruiting $\mathrm{HDAC} 1$, thus defining a novel mechanism controlling TGF- $\beta$ signaling.

Dr. Chai JiJie applies X-ray crystallography and biochemical characterization on the plant receptor-like kinases (RLKs) and the NOD-type receptors ( $\mathrm{R}$ proteins in plants and NLRs in animals). The current study [15] reports the crystal structure of the extracellular LRR domain of RPK2 containing two island domain from Arabidopsis, which reveals a potential ligand binding site at the C-terminal side of RPK2-LRR. This result provides insight into RPK2mediated signaling.

Research from Dr. Xie DaoXin's laboratory centers on general understanding of plant hormone signaling, with a keen focus on jasmonate perception and signal transduction. His work reported in this issue [16] presents genetic, physiological and biochemical evidence to demonstrate that mutations of key amino acids, such as GLY98, LEU245 and GLU543, in the jasmonate receptor COI1 exhibit distinct impact on male fertility and temperature sensitivity in Arabidopsis.

1 Shen R, Cai LW, Meng DC, Wu LP, Guo K, Dong GX, Liu L, Chen JC, Wu Q, Chen GQ. Benzene containing polyhydroxyalkanoates homo- and copolymers synthesized by genome edited Pseudomonas entomophila. Sci China Life Sci, 2014, 57: 4-10

2 Zhang XF, Jiang T, Yu YT, Wu Z, Jiang SC, Lu K, Feng XJ, Liang S, Lu YF, Wang XF, Zhang DP. Arabidopsis co-chaperonin CPN20 antagonizes $\mathrm{Mg}$-chelatase $\mathrm{H}$ subunit to derepress ABA-responsive WRKY40 transcription repressor. Sci China Life Sci, 2014, 57: $11-21$

3 Yang YC, Umetsu J, Lu ZJ. Global signatures of protein binding on structured RNAs in Saccharomyces cerevisiae. Sci China Life Sci, 2014, 57: 22-35

4 Li JR, Wu Y, Qi YJ. microRNAs in a multicellular green alga Volvox carteri. Sci China Life Sci, 2014, 57: 36-45
5 Lu BY, Shao LS, Feng SX, Wang T, Zhong Y. The $\beta$-alanylmonoamine synthase ebony is regulated by schizophrenia susceptibility gene dysbindin in Drosophila. Sci China Life Sci, 2014, 57: 46-51

6 Li YX, Liu XN, Wang XW, Zhang XG. Sequence signatures of genes with accompanying antisense transcripts in Saccharomyces cerevisiae. Sci China Life Sci, 2014, 57: 52-58

7 Peng JY, Zhang R, Cui YT, Liu HD, Zhao XX, Huang L, Hu MX, Yuan XX, Ma BY, Ma XW, Takashi U, Masaaki K, Liang XJ, Yu L. Atg5 regulates late endosome and lysosome biogenesis. Sci China Life Sci, 2014, 57: 59-68

8 Ren J, Wang J, Wang ZX, Wu JW. Structural and biochemical insights into the homotypic $\mathrm{PB} 1-\mathrm{PB} 1$ complex between $\mathrm{PKC} \zeta$ and $\mathrm{p} 62$. Sci China Life Sci, 2014, 57: 69-80

9 Wu LY, Lu PW, Ma WW, Chu CC, Xu HP, Qi H. Identification of a new isoform of the murine Sh2d1a gene and its functional implications. Sci China Life Sci, 2014, 57: 81-87

10 Guo DX, Shi XL, Song DK, Zhang LQ. Persistence of VRC01-resistant HIV-1 during antiretroviral therapy. Sci China Life Sci, 2014, 57: 88-96

11 Mei KR, Jin Z, Ren FL, Wang YY, Chang ZJ, Wang XQ. Structural basis for the recognition of RNA polymerase II C-terminal domain by CREPT and p15RS. Sci China Life Sci, 2014, 57: 97-106

12 Wu LZ, Zhou LK, Chen C, Gong JY, Xu L, Ye J, Li D, Li P. Cidea controls lipid droplet fusion and lipid storage in brown and white adipose tissue. Sci China Life Sci, 2014, 57:107-116

13 Yu Y, Tong P, Li Y, Lu ZF, Chen YH. 10E8-like neutralizing antibodies against HIV-1 induced using a precisely designed conformational peptide as a vaccine prime. Sci China Life Sci, 2014, 57: $117-127$

14 Yan XH, Pan J, Xiong WW, Cheng MZ, Sun YY, Zhang SP, Chen YG. Yin Yang 1 (YY1) synergizes with Smad7 to inhibit TGF- $\beta$ signaling in the nucleus. Sci China Life Sci, 2014, 57: 128-136

15 Song W, Han ZF, Sun YD, Chai JJ. Crystal structure of a plant leucine rich repeat protein with two island domains. Sci China Life Sci, 2014, 57: 137-144

16 Huang H, Wang CL, Tian HX, Sun Y, Xie DX, Song SS. Amino acid substitutions of GLY98, LEU245 and GLU543 in COI1 distinctively affect jasmonate-regulated male fertility in Arabidopsis. Sci China Life Sci, 2014, 57: 145-154

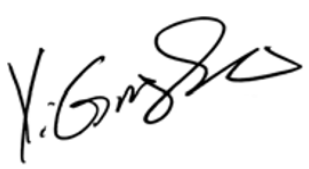

Professor and Dean School of Life Sciences Tsinghua University November 15th, 2013

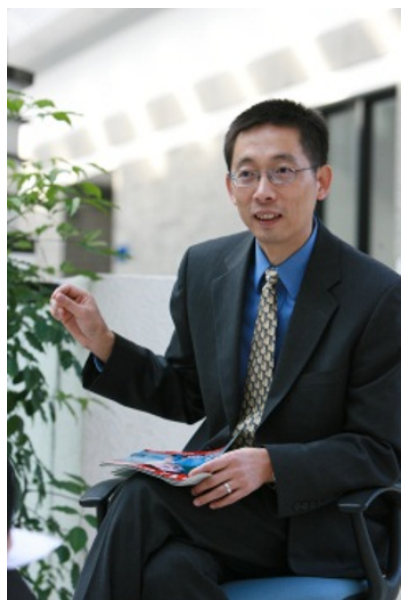

Open Access This article is distributed under the terms of the Creative Commons Attribution License which permits any use, distribution, and reproduction in any medium, provided the original author(s) and source are credited. 